\title{
Predicting the Appearance of Materials Using Lorenz-Mie Theory
}

\author{
Frisvad, Jeppe Revall; Christensen, Niels Jørgen; Jensen, Henrik Wann
}

Published in:

The Mie Theory

Link to article, DOI:

10.1007/978-3-642-28738-1_4

Publication date:

2012

Link back to DTU Orbit

Citation (APA):

Frisvad, J. R., Christensen, N. J., \& Jensen, H. W. (2012). Predicting the Appearance of Materials Using LorenzMie Theory. In The Mie Theory: Basics and Applications (pp. 101-133). Springer. Springer Series in Optical Sciences No. 169 https://doi.org/10.1007/978-3-642-28738-1_4

\section{General rights}

Copyright and moral rights for the publications made accessible in the public portal are retained by the authors and/or other copyright owners and it is a condition of accessing publications that users recognise and abide by the legal requirements associated with these rights.

- Users may download and print one copy of any publication from the public portal for the purpose of private study or research.

- You may not further distribute the material or use it for any profit-making activity or commercial gain

- You may freely distribute the URL identifying the publication in the public portal

If you believe that this document breaches copyright please contact us providing details, and we will remove access to the work immediately and investigate your claim. 


\title{
Chapter 4 \\ Predicting the Appearance of Materials Using Lorenz-Mie Theory
}

\author{
Jeppe Revall Frisvad, Niels Jørgen Christensen and Henrik Wann Jensen
}

Computer graphics systems today are able to produce highly realistic images. The realism has reached a level where an observer has difficulties telling whether an image is real or synthetic. The exception is when we try to compute a picture of a scene that really exists and compare the result to a photograph of the real scene. In this direct comparison, an observer quickly identifies the synthetic image. One of the problems is to model all the small geometrical details correctly. This is a problem that we will not consider. But even if we pick a simple experimental setup, where the objects in the scene have few geometrical details, a graphics system will still have a hard time predicting the result of taking a picture with a digital camera. The problem here is to model the optical properties of the materials correctly. In this paper, we show how Lorenz-Mie theory enables us to compute the optical properties of turbid materials such that we can predict their appearance. To describe the entire process of predicting the appearance of a material, we include a description of the mathematical models used in realistic image synthesis.

\subsection{Introduction}

The appearance of an object is a result of light reaching the eye after interacting with matter. One way to capture the appearance of an object is to take a picture of it. This means that a digital camera is not only an inexpensive consumer product, it is also a fairly advanced device for measuring light. As we know from spectroscopy, light can tell us a great deal about the material that it is illuminating. The question is then: what can we learn about a material by taking a picture of it using a digital camera? The

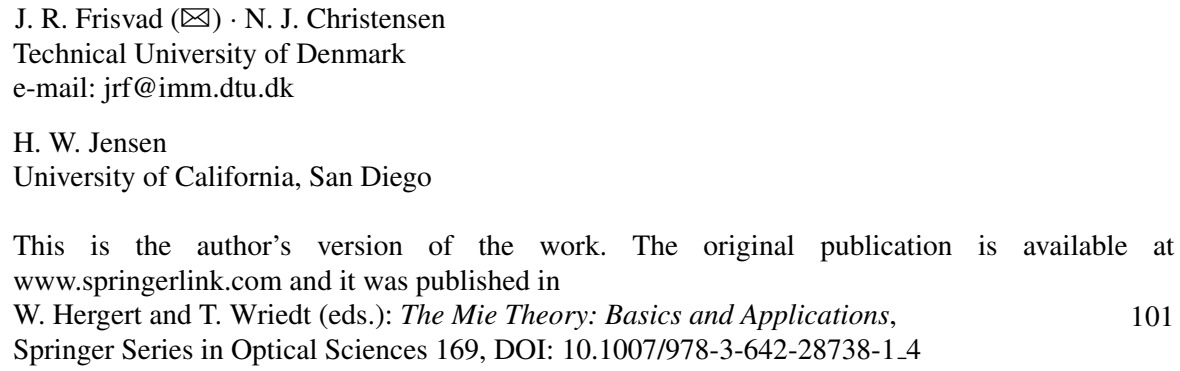


immediate response is: not a lot. Unless we have a lot of pictures of similar materials in the same lighting environment which we can use to make a statistical model (this is image analysis). Or unless we have a physical model of the material and lighting environment that we can use to predict the picture (this is computer graphics). The purpose of this paper is to show how to construct a model which predicts the result of taking a picture of a material in a controlled lighting environment. In short, we describe how to predict the appearance of materials. Once we have an appearance model that predicts the result of taking a picture of a material, we can take a picture of a similar material and fit the parameters in the model such that the picture of this similar material is obtained. In this way, we can use a picture of a material to estimate the properties of the material (analysis by synthesis).

The advantage of using a camera instead of a spectrometer is that it is inexpensive and that it does not require small samples to be prepared for analysis inside a larger device. Taking a picture using a digital camera is a simple, non-invasive process. This means that it is particularly useful for food quality control and medical diagnosis.

To construct an appearance model that predicts the result of taking a picture of a material, we need (a) a model of the camera, (b) a geometrical model of the material sample, (c) a model of the light sources, (d) a model for light propagation, and (e) a model for light scattering. The problematic model is the one for light scattering because the material properties are unknown. The macroscopic optical properties of materials are so diverse that it is impossible to measure and tabulate the properties of all materials directly. This is where Lorenz-Mie theory comes in handy. LorenzMie theory provides the link between the particle composition of a material and its macroscopic optical properties. The particle composition of a material is a very flexible starting point. There are many different ways to combine small particles into one macroscopic bulk material. To limit the potentially large number of input parameters, we use empirical models to describe the size distribution and optical properties of the particles. When available, these empirical models enable us to construct material appearance models that depend on only few essential inputs. A list of ingredients, for example, where the relative contents are specified in weight percent. The example used in this chapter is cow's milk with fat and protein contents of the milk (in weight percent) as input parameters.

\subsection{Realistic Image Synthesis}

The development of models for computing realistic images is a branch of computer graphics known as realistic image synthesis $[1,2]$. This field of research provides the link between physical models of light and materials and the process of computing an image as if it were taken with a real digital camera or as if it were seen with the eyes of a human observer. Light transport and scattering is modelled in realistic image synthesis using the mathematical model developed for radiative transfer [3, 4]. The process of generating an image using a computer is referred to as rendering. Thus realistic image synthesis is also known as realistic rendering. 
The basics of realistic image synthesis have been described in many text books. A useful reference which provides both detailed descriptions and source code is the book by Pharr and Humphreys [5]. The following subsections provide brief descriptions of the five models that we need.

\subsubsection{Camera}

Think about taking a picture. The first thing you do is to position the camera and orient it toward the objects that you wish to photograph. To model this setup, we need a camera position $\boldsymbol{p}$, a viewing direction $\mathbf{v}$, and an up-vector $\mathbf{u}$. A digital camera is built around a photoactive charge-coupled device chip (CCD chip) which has a limited number of bins across its area. This means that output images from a digital camera have a specific resolution (image width $W$ and height $H$ ) measured in number of pixels. Each pixel corresponds to a small area of the CCD chip and it has an associated vector which describes the amount of light (of different colours) that reached this area during exposure. To capture an image is to expose the chip for a short amount of time and record the vectors for all the pixels. We model the light sensitive area of the CCD chip by a rectangle in the image plane, and we will call it the film. The viewing direction $\mathbf{v}$ is normal to the image plane and the up direction of the image plane is given by the up-vector $\mathbf{u}$. To position the film in space, we centre it around the camera position $\boldsymbol{p}$ and move it a distance $d$ in the viewing direction. The distance $d$ depends on the lens system of the camera and is called the camera constant. The size of the film (width by height, $w \times h$ ) is usually specified by an angle in the vertical direction called the field of view, fov, and an aspect ratio $a$ such that

$$
\begin{aligned}
w & =a h \\
h & =2 d \tan (\text { fov } / 2)
\end{aligned}
$$

Using the camera resolution $(W \times H)$, we can divide the film into pixels. To compute an image, we trace a number of rays from $\boldsymbol{p}$ through each pixel of the film.

\subsubsection{Geometry of Objects}

Representation of arbitrarily shaped objects is a difficult problem with many solutions. The computer graphics community is with little doubt the most extensive user of surface models. The mainstream approach is to use a large number of small triangles. If we let some triangles have common edges with others, we obtain a triangle mesh. If there are no holes in the mesh, it represents the surface of a volume which could be an object in our scene. 
The triangle mesh poses some problems. Unless we want to make objects with sharp edges, we need an awfully large number of triangles to make an object look smooth. Even more triangles are needed if we want smooth reflections and refractions as well. This problem is usually solved by computing a normal for each vertex in the triangle mesh as originally suggested by Phong [6]. Such a vertex normal is an average of the normals associated with the neighbouring triangle faces. When we want to find the surface normal where a ray intersects a triangle, we interpolate the vertex normals across the triangle using trilinear interpolation. In this way, we have a number of points (the vertices in the triangle mesh) which make out a discrete representation of a smooth surface.

\subsubsection{Light Sources}

A light source is typically modelled by letting an object in the scene emit light equally in all directions from every surface point. Another option is to have a spherical map around the scene which acts as a background source or environment lighting. This is called an environment map and it is modelled as if it were infinitely far away. It is particularly useful for outdoor sceneries, where one can use a model of the atmosphere to compute a realistic environment map [7, 8, 9].

Another important type of light source is laser. As has been discovered in biomedical optics [10], shining a laser into a turbid material (skin, for example) tells us surprisingly much about the material. To model a laser source, we use a circular disk which emits light weighted by a Gaussian function diminishing with the distance to the centre of the disk. Laser is also nearly collimated. This is modelled by letting the source emit light only in the direction normal to the surface of the disk.

\subsubsection{Light Propagation}

Pictures most often capture light in the visible part of the electromagnetic spectrum. Visible wavelengths are very short, ranging from $380 \mathrm{~nm}$ to $780 \mathrm{~nm}$, and it was shown by Sommerfeld and Runge [11] that plane electromagnetic waves propagating in a non-absorbing, homogeneous dielectric are equivalent to rays of light for $\lambda \rightarrow 0$, where $\lambda$ denotes the wavelength in vacuum. This means that rays of light are often a good model of visible light. According to Fermat's principle, rays of light follow the path of least time [12, pp. 457-463]. We can use this principle to trace rays of light through a digitally modelled scene. The main limitation is that wave phenomena such as interference and diffraction will not be captured. The visual effects from such phenomena must then be incorporated into the scattering properties of the materials in the scene. Diffraction by particles is incorporated into the macroscopic scattering properties of a material using Lorenz-Mie theory. This is covered in Section 4.2.5. Interference and diffraction effects caused by microfacets in the surface of an object 
are incorporated into surface reflection models. We will not discuss microfacetted surface reflection models.

Rays of light are traced in straight lines as long as they travel in a homogeneous medium, since, in this case, the shortest path is also the path of least time. Having settled on a ray theory of light, we trace light backwards from the camera position $\boldsymbol{p}$ through a pixel into our scene to see if it intersects one of the triangles that the objects are composed of. The number of triangles is large, so some kind of spatial data structure is needed to find the triangles that are most likely to be intersected $[13,14,15,16]$. An efficient ray-triangle intersection algorithm has been developed by Möller and Trumbore [17]. Only the closest intersected triangle is considered, other intersected triangles are hidden behind it. Once a point of intersection is identified and a surface normal has been computed, the next step is to estimate the light that reaches this point. Assuming a smooth surface, light reflects off the surface and refracts into the material as described by Fresnel's formulae for reflectance [18]. New rays are traced from the point of intersection in the directions of reflection and refraction (as dictated by the laws of reflection and refraction). Light reflects and refracts recursively until it reaches a light source. This is called ray tracing, and this recursive version, where new rays are traced from the point of intersection, was first described for graphics by Whitted [19]. If light refracts into a turbid material, it scatters as described in Section 4.2.5.

What we trace along a ray is electromagnetic energy. The rays follow the direction of the time-averaged Poynting vector as closely as possible.

\subsubsection{Light Scattering}

In the previous section, we found a way to trace energy through matter. This is all we need to render materials with a continuous interior. However, if a material is composed of millions of microscopic particles, it is infeasible to model the surface of every particle. Therefore we introduce a model for macroscopic scattering. The model used to describe light scattering is the radiative transfer equation [3]:

$$
(\omega \cdot \nabla) L(\boldsymbol{x}, \omega)=-\sigma_{t}(\boldsymbol{x}) L(\boldsymbol{x}, \omega)+\sigma_{s}(\boldsymbol{x}) \int_{4 \pi} p\left(\boldsymbol{x}, \omega^{\prime}, \omega\right) L\left(\boldsymbol{x}, \omega^{\prime}\right) \mathrm{d} \omega^{\prime}+L_{e}(\boldsymbol{x}, \omega),
$$

where $L(\boldsymbol{x}, \omega)$ is the radiance at $\boldsymbol{x}$ in the direction $\omega$, the subscript $e$ denotes emission, and $\sigma_{s}, \sigma_{a}$, and $\sigma_{t}=\sigma_{s}+\sigma_{a}$ are the scattering, absorption, and extinction coefficients respectively. The phase function $p$ specifies the normalised distribution of the scattered light. Radiance is a radiometric quantity measured in energy flux per solid angle per projected area. The equation splits the directional derivative (lefthand side), that is, the change in radiance along a ray, into three terms (right-hand side): The first term denotes the exponential attenuation, the second denotes the inscattering from all directions, and the third is an emission term. 
The radiometric quantity radiance is fundamental in radiative transfer. It is what the radiative transfer equation (4.1) is concerned with and it is defined (radiometrically) by

$$
L=\frac{\mathrm{d}^{3} Q}{\mathrm{~d} t \omega \mathrm{d} A_{\perp}}=\frac{\mathrm{d}^{2} \Phi}{\mathrm{d} \omega \mathrm{d} A \cos \theta},
$$

where $Q$ is energy, $t$ is time, $\Phi=\mathrm{d} Q / \mathrm{d} t$ is energy flux, $\omega$ is solid angle, $A_{\perp}$ is projected area, $A$ is area, and $\theta$ is the angle between the normal to the area $\mathrm{d} A$ and the considered direction. Radiance describes the flow of energy through a differential area $\mathrm{d} A$. The energy flows in a directional differential volume $\mathrm{d} \omega$ which is not necessarily normal to the area. The purpose of radiance is thus to describe the energy flow in a ray of light incident on a surface.

Since radiance is a quantity based on energy, we assume that it follows the flow of energy through a medium. Thus we trace rays using Fermat's principle. However, we have to be careful and take into account that radiance denotes flux per solid angle per projected area. When we follow a ray of light through a material, we have to take into account that the radiance may change along the ray. Let us see how radiance changes upon refraction.

Consider a ray of light in a medium with refractive index $n_{1}=n_{1}^{\prime}+i n_{1}^{\prime \prime}$ incident on a surface patch $\mathrm{d} A$ of a medium with refractive index $n_{2}=n_{2}^{\prime}+i n_{2}^{\prime \prime}$. Due to energy conservation at the boundary, the following condition must hold:

$$
L_{i} \cos \theta_{i} \mathrm{~d} A \mathrm{~d} \omega_{i}=L_{r} \cos \theta_{r} \mathrm{~d} A \mathrm{~d} \omega_{r}+L_{t} \cos \theta_{t} \mathrm{~d} A \mathrm{~d} \omega_{t},
$$

where $L_{i}, L_{r}$, and $L_{t}$ are the incident, reflected, and transmitted radiances and likewise $\theta_{i}, \theta_{r}$, and $\theta_{t}$ are the angles of incidence, reflection, and refraction. In spherical coordinates, the solid angles are defined by

$$
\begin{aligned}
\mathrm{d} \omega_{i} & =\sin \theta_{i} \mathrm{~d} \theta_{i} \mathrm{~d} \phi_{i} \\
\mathrm{~d} \omega_{r} & =\sin \theta_{r} \mathrm{~d} \theta_{r} \mathrm{~d} \phi_{r} \\
\mathrm{~d} \omega_{t} & =\sin \theta_{t} \mathrm{~d} \theta_{t} \mathrm{~d} \phi_{t} .
\end{aligned}
$$

Using the law of reflection $\theta_{i}=\theta_{r}$ and the fact that both the reflected and transmitted rays lie in the plane of incidence $\phi_{i}=\phi_{r}=\phi_{t}$, the boundary condition becomes

$$
L_{i} \cos \theta_{i} \sin \theta_{i} \mathrm{~d} \theta_{i}=L_{r} \cos \theta_{i} \sin \theta_{i} \mathrm{~d} \theta_{i}+L_{t} \cos \theta_{t} \sin \theta_{t} \mathrm{~d} \theta_{t}
$$

To find the transmitted angle, we have to consider the direction of the transmitted ray. This is given by the law of refraction. We find

$$
\begin{aligned}
\sin \theta_{t} & =\frac{n_{1}^{\prime}}{n_{2}^{\prime}} \sin \theta_{i} \\
\cos \theta_{t} \mathrm{~d} \theta_{t} & =\frac{n_{1}^{\prime}}{n_{2}^{\prime}} \cos \theta_{i} \mathrm{~d} \theta_{i} .
\end{aligned}
$$


With this result, the boundary condition is $L_{i}=L_{r}+\left(\frac{n_{1}^{\prime}}{n_{2}^{\prime}}\right)^{2} L_{t}$. Or, in terms of outgoing radiance,

$$
L_{o}=R L_{i}+(1-R)\left(\frac{n_{1}^{\prime}}{n_{2}^{\prime}}\right)^{2} L_{i}
$$

where $R$ is the Fresnel reflectance.

These equations show that radiance is not constant along a ray of light. If we move along a ray through a heterogeneous medium, the radiance should be modified. Generalizing the result above to the interior of a medium, we approximately have $[20,21]$

$$
\frac{L_{1}}{n_{1}^{\prime 2}} \mathrm{~d} A_{1}=\frac{L_{2}}{n_{2}^{\prime 2}} \mathrm{~d} A_{2}
$$

where the subscripts 1 and 2 denote two locations along a ray of light. This means that the quantity $L_{1} / n_{1}^{\prime 2}$ is (approximately) constant along the ray. If we store $L_{1} / n_{1}^{\prime 2}$ before tracing a ray from one point in a medium, then the radiance at the destination point is $L_{2}=n_{2}^{\prime 2} L_{1} / n_{1}^{\prime 2}$. Now that we know how radiance behaves as we move along a ray, we are ready to evaluate the radiative transfer equation (4.1) using ray tracing.

Rendering realistic images using the radiative transfer equation, was first proposed by Kajiya and Von Herzen [22]. Most rendering algorithms use approximate evaluation schemes to gain speed. Here we will only describe the general way of evaluating the radiative transfer equation. The remainder of this section is a short account of Monte Carlo path tracing, which is a sampling-based rendering algorithm that works in general. More information is available in the book by Pharr and Humphreys [5]. Unfortunately, Pharr and Humphreys stop their treatment at single scattering. Evaluation of the general case has been described by Pattanaik and Mudur [23].

The emission term in the radiative transfer equation is just an added constant. It is not difficult to include it, but it makes the equations rather long. Therefore we leave out the emission term in the following. The general approach is as follows. We first parameterise the radiative transfer equation using the distance $s^{\prime}$ that light has traveled along a path into a medium. We have (for a non-emitter):

$$
\frac{\mathrm{d} L\left(s^{\prime}\right)}{\mathrm{d} s^{\prime}}+\sigma_{t}\left(s^{\prime}\right) L\left(s^{\prime}\right)=\sigma_{s}\left(s^{\prime}\right) \int_{4 \pi} p\left(s^{\prime}, \omega^{\prime}, \omega\right) L\left(s^{\prime}, \omega^{\prime}\right) \mathrm{d} \omega^{\prime},
$$

where $\omega$ denotes the tangential direction of the path at the distance $s^{\prime}$ along the path.

The parameterised equation (4.3) is a linear, first-order, ordinary differential equation (where $\sigma_{t}\left(s^{\prime}\right)$ is a variable coefficient). One way to solve such an equation is by means of an integration factor:

$$
T_{r}\left(s^{\prime}, s\right)=\exp \left(-\int_{s^{\prime}}^{s} \sigma_{t}(t) \mathrm{d} t\right) .
$$

With this factor, the equation (4.3) becomes 


$$
\frac{\mathrm{d}}{\mathrm{d} s^{\prime}}\left(T_{r}\left(s^{\prime}, s\right) L\left(s^{\prime}\right)\right)=T_{r}\left(s^{\prime}, s\right) \sigma_{s}\left(s^{\prime}\right) \int_{4 \pi} p\left(s^{\prime}, \omega^{\prime}, \omega\right) L\left(s^{\prime}, \omega^{\prime}\right) \mathrm{d} \omega^{\prime} .
$$

Note that $T_{r}(s, s)=1$. Then by integration along the ray from the surface $s^{\prime}=0$ to the considered location in the medium $s^{\prime}=s$, the equation (4.5) attains the form:

$$
L(s)=T_{r}(0, s) L(0)+\int_{0}^{s} T_{r}\left(s^{\prime}, s\right) \sigma_{s}\left(s^{\prime}\right) \int_{4 \pi} p\left(s^{\prime}, \omega^{\prime}, \omega\right) L\left(s^{\prime}, \omega^{\prime}\right) \mathrm{d} \omega^{\prime} \mathrm{d} s^{\prime} .
$$

For convenience some of the different mathematical quantities encountered in this derivation have been given appropriate names in radiative transfer theory [3]. The distance $s$ (or $s^{\prime}$ ) traveled along the ray inside the medium, is referred to as the depth. The integral in Equation 4.4 is called the optical thickness and is denoted by the symbol $\tau$, that is,

$$
\tau\left(s^{\prime}, s\right)=\int_{s^{\prime}}^{s} \sigma_{t}(t) \mathrm{d} t
$$

The integration factor itself $T_{r}\left(s^{\prime}, s\right)=e^{-\tau\left(s^{\prime}, s\right)}$ is sometimes referred to as the beam (or path) transmittance. Finally, the first term on the right hand side of the formal solution (4.6) for the radiative transfer equation (4.1) is referred to as the direct transmission term whereas the second term is called the diffusion term. Realistic rendering is all about evaluating these terms in various kinds of ways.

To evaluate the equation (4.6) using Monte Carlo path tracing, we first take a look at the direct transmission term $T_{r}(0, s) L(0)$. For this term we need to estimate the value of the optical thickness $\tau(0, s)$. If the medium is homogeneous, this optical thickness is simply given by

$$
\tau(0, s)=\sigma_{t} s
$$

For heterogeneous media, we use sampling. The optical thickness is found quite efficiently by the estimator:

$$
\sum_{i=0}^{N-1} \sigma_{t}\left(t_{i}\right) \Delta t
$$

where $\Delta t$ is the step size (given as user input) and $t_{i}$ are locations along the ray found using a single uniform random variable $\xi \in[0,1]$ :

$$
t_{i}=\frac{\xi+i}{N} s
$$

The number $N$ of locations along the ray is found using the depth $s$ which is the distance to the next surface, that is, $N=\lfloor s / \Delta t\rfloor$. Having estimated the optical thickness $\tau(0, s)$, the beam transmittance $T_{r}(0, s)$ is easily found and multiplied by the amount of radiance $L(0)$ to reveal the direct transmission term. The radiance $L(0)$ is the radiance which contributes to the ray at the surface of the medium. 
Evaluating the diffusion term is more involved. What we need is an estimator of the form

$$
\frac{1}{N} \sum_{j=0}^{N-1} \frac{T_{r}\left(s_{j}^{\prime}, s\right) \sigma_{s}\left(s_{j}^{\prime}\right) J\left(s_{j}^{\prime}\right)}{\operatorname{pdf}\left(s_{j}^{\prime}\right)},
$$

where the probability distribution function (pdf) preferably cancels out the transmittance. The source function

$$
J\left(s^{\prime}\right)=\int_{4 \pi} p\left(s^{\prime}, \omega^{\prime}, \omega\right) L\left(s^{\prime}, \omega^{\prime}\right) \mathrm{d} \omega^{\prime}
$$

is evaluated using a distribution of samples over the entire unit sphere.

The following pdf is a good choice for the diffusion term estimator (4.10):

$$
\operatorname{pdf}\left(s_{j}^{\prime}\right)=\sigma_{t}\left(s_{j}^{\prime}\right) T_{r}\left(s_{j}^{\prime}, s\right),
$$

since it has the simple complementary cumulative distribution function

$$
\operatorname{ccdf}\left(s_{j}^{\prime}\right)=\int_{s_{j}^{\prime}}^{s} \sigma_{t}(t) T_{r}(t, s) \mathrm{d} t=T_{r}(s, s)-T_{r}\left(s_{j}^{\prime}, s\right)=1-T_{r}\left(s_{j}^{\prime}, s\right) .
$$

Using the inverse transformation method [5, e.g.], we get the following equation for sampling an interaction along the ray according to this pdf:

$$
\xi_{j}=1-T_{r}\left(s_{j}^{\prime}, s\right)=1-e^{-\tau\left(s_{j}^{\prime}, s\right)} \quad \text { or } \ln \left(\xi_{j}\right)+\tau\left(s_{j}^{\prime}, s\right)=0,
$$

where $\xi_{j} \in[0,1]$ is a uniform random variable for sample $j$. An interaction is either scattering according to the source function (4.11) or absorption. The depth of the sample is easily found for homogeneous media, where we have $\tau\left(s_{j}^{\prime}, s\right)=\left(s-s_{j}^{\prime}\right) \sigma_{t}$, which gives

$$
s_{j}^{\prime}=s+\frac{\ln \left(\xi_{j}\right)}{\sigma_{t}} .
$$

If $s_{j}^{\prime}<0$, there is no interaction for sample $j$. For heterogeneous media we have to step along the ray to find out where the optical thickness matches the event. Starting at $t_{1}$ in $t_{i}=s-i \Delta t$, we step along the ray by incrementing $i$, and if $\ln \left(\xi_{j}\right)+\tau\left(t_{i}, s\right)>0$, we stop and compute the location of the interaction as follows [23]:

$$
s_{j}^{\prime}=s-(i-1) \Delta t+\frac{\tau\left(t_{i-1}, s\right)+\ln \left(\xi_{j}\right)}{\sigma_{t}\left(t_{i}\right)} .
$$

Here it is assumed that $\sigma_{t}\left(t_{i}\right)$ is approximately constant in steps of size $\Delta t$ along the ray, such that $\sigma_{t}\left(s_{j}^{\prime}\right) \approx \sigma_{t}\left(t_{i}\right)$. If we end up with $t_{i} \leq 0$ before the other criteria is fulfilled, there is no interaction for sample $j$. The optical thicknesses needed in order to find $s_{j}^{\prime}$ are evaluated in the same way as when we evaluated $\tau(0, s)$ only with $s-t_{i}$ in Equation 4.9 instead of $s$. 
Finally, the scattering coefficient $\sigma_{s}\left(s_{j}^{\prime}\right)$ in the estimator (4.10) and the extinction coefficient $\sigma_{t}\left(s_{j}^{\prime}\right)$ in the probability distribution function (pdf) are canceled out by means of a Russian roulette. At every interaction a Russian roulette is carried out using the scattering albedo, which is defined by

$$
\alpha\left(s_{j}^{\prime}\right)=\sigma_{s}\left(s_{j}^{\prime}\right) / \sigma_{t}\left(s_{j}^{\prime}\right),
$$

as the probability of a scattering event.

Using the sampling scheme described above, the estimator (4.10) becomes:

$$
\frac{1}{N} \sum_{j=0}^{N-1} J\left(s_{j}^{\prime}\right)=\frac{1}{N M} \sum_{j=0}^{N-1} \sum_{k=0}^{M-1} \frac{p\left(s_{j}^{\prime}, \omega_{k}^{\prime}, \omega\right) L\left(s_{j}^{\prime}, \omega_{k}^{\prime}\right)}{\operatorname{pdf}\left(\omega_{k}^{\prime}\right)}
$$

for $\xi<\alpha\left(s_{j}^{\prime}\right)$ and 0 otherwise. For an isotropic phase function, sampling a uniform distribution over the unit sphere leaves only $L\left(s_{j}^{\prime}, \omega_{k}^{\prime}\right)$ in the sum.

To summarise the algorithm, a ray is traced from an observer through a scene, when it refracts into a turbid material, we do the following:

1. Trace a refracted ray. The radiance carried along the refracted ray is corrected according to the boundary condition (Equation 4.2).

2. The tracing of the refracted ray gives the depth $s$ to the next surface.

3 . The radiance $L(0)$ which contributes to the ray at the surface is found by tracing new rays in the directions of reflection and refraction. If $L(0)$ is not too small, we evaluate the direct transmission term:

a. The optical thickness $\tau(0, s)$ is estimated using Equations 4.9 and 4.8 (or Equation 4.7 for homogeneous media).

b. The direct transmission term $T_{r}(0, s) L(0)$ is found using the optical thickness $\tau(0, s)$ (see Equation 4.4) and the radiance which contributes to the ray at the surface $L(0)$.

4. For every diffusion term sample $j=1, \ldots, N$, a sample depth $s_{j}^{\prime}$ is found using Equation 4.13 (or Equation 4.12 for homogeneous media).

5. For the samples $s_{j}^{\prime}>0$, a Russian roulette is done using the scattering albedo $\alpha\left(s_{j}^{\prime}\right)$. For $\xi<\alpha\left(s_{j}^{\prime}\right)$, where $\xi \in[0,1[$ is a random variable, there is a scattering event.

6. For every scattering event, the phase function $p\left(s_{j}^{\prime}, \omega_{k}^{\prime}, \omega\right)$ is evaluated in $M$ sampled directions $\omega_{k}^{\prime}$ with $k=1, \ldots, M$. Likewise $M$ new rays are traced at the position $s_{j}^{\prime}$ in the directions $\omega_{k}^{\prime}$ to obtain the radiances $L\left(s_{j}^{\prime}, \omega_{k}\right)$. Using Equation 4.14 this gives an estimate of the diffusion term.

7. Finally, the direct transmission term and the diffusion term are added to get the radiance emergent at the surface $L(s)$.

The numbers of samples chosen are often $N=1$ and $M=1$. Then we get a very noisy sample image rather quickly. Another sample is then rendered and this is averaged with the previous one. The next sample is weighted by one third and added to the other two samples which are weighted by two thirds and so on. In this way the image will improve itself over time. Even so, the Monte Carlo path tracing procedure 
spawns a formidable number of rays. It is very slow, but it converges to the intended result in an unbiased manner. This is nice in a predictive rendering context, where we want to predict the appearance of real-world materials.

\subsection{Predicting Appearance}

In order to predict the result of taking a picture with a digital camera, we need to digitally model the 3D scenery that is going to be in the picture as explained in Section 4.2.2. For example, in order to compute a realistic image of a glass of milk, we need a geometric model of the glass and the milk inside it. The most practical way of modelling such geometry is to use a computer aided design (CAD) system (e.g. AutoCAD ${ }^{\circledast}$ or Pro/ENGINEER ${ }^{\circledast}$ ) or a 3D modelling system (e.g. 3ds Max $^{\circledast}$, Softimage ${ }^{\circledast}$, or Blender $\left.{ }^{\text {rM }}\right)$. A scene modelled using one of these systems can be exported to a text file and imported into a rendering system which implements a camera model (see Section 4.2.1) and the models for light propagation and scattering described in Sections 4.2.4 and 4.2.5. This could be our own rendering system or another physically-based rendering system such as Maxwell Render ${ }^{\mathrm{TM}}$ or Indigo Renderer. Once the geometry of the scene is in place, we need information about the materials and the light sources in the scene. More specifically, we need the optical properties of the materials and the emission profiles of the light sources.

To acquire the optical properties of a material, one option is to measure them. Another option is to compute them from the particle contents of the material. The second option is made possible by the Lorenz-Mie theory, and it provides us with a very flexible way of modelling how the appearance of a material changes when we change its contents. Nevertheless, the Lorenz-Mie theory has only reluctantly been adopted in graphics. In the two papers [24, 25] where the theory was first considered for graphics applications, it was found to be either too complicated [24] or too restricted [25] to be useful. The problematic restrictions are that the material should consist of nearly spherical particles embedded in a non-absorbing host medium. This significantly limits the number of materials that can be modelled by the original version of the theory. Even so, the original theory has proven useful for modelling the appearance of some special materials: Callet [26] used the theory to model pigmented materials (such as paints, plastics, inks, and cosmetics which consist of pigmented particles in a transparent solvent). Jackèl and Walter [8] used it to model the atmosphere and rainbows. Nishita and Dobashi [27] used the theory to model various other materials consisting of particles in air (clouds, smoke/gas, fog/haze, snow, and sand). Most recently, we have seen a development towards generalisation of the Lorenz-Mie theory such that it becomes useful for a wider range of materials. Such new developments were adapted for graphics by Frisvad et al. [28].

In the following section, we describe the link between material contents and macroscopic optical properties of the material as provided by the Lorenz-Mie theory. 


\subsubsection{Computing Optical Properties}

The input parameters for the radiative transfer equation (4.1) are the phase function $p\left(\boldsymbol{x}, \omega^{\prime}, \omega\right)$, the scattering coefficient $\sigma_{s}(\boldsymbol{x})$, and the extinction coefficient $\sigma_{t}(\boldsymbol{x})$ (or the absorption coefficient since $\left.\sigma_{t}=\sigma_{a}+\sigma_{s}\right)$. Together with the index of refraction $n_{\text {bulk }}(\boldsymbol{x})$, these parameters constitute the optical properties of a material. In the following, we will omit the dependency on the position $x$ in the material. Then we just have to remember that this dependency should be inserted if the material is heterogeneous.

The phase function and the scattering coefficient are collectively referred to as the scattering properties of the material. The direction of the incoming light $\omega^{\prime}$ is called the forward direction while $\omega$ is the direction of the scattered light. The plane spanned by these two vectors is called the scattering plane and the angle between them $\theta$ is called the scattering angle.

Scattering under the surface of a material is typically caused by particles. For simplicity, let us assume that the particles are small, randomly distributed throughout the material, not too densely packed, and approximately spherical. A particle is considered small when it is not directly visible to the human eye from the distance that it is observed. If every particle were visible, we would have to model the surface of each individual particle. If the particles were not randomly distributed, we would again need to make a very precise model taking into account the ordered placement of the particles in the material. When we assume that the particles are not too densely packed, we assume that the distance between them is considerably larger than the wavelength of the light. Under this assumption, we say that the particles scatter light independently of each other. The assumption about approximately spherical particles ensures that scattering is symmetric around the forward direction and that the two polarisation components do not affect each other. In other words, we only need two scattering components to describe the scattering of a spherical particle: $S_{1}(\theta)$ for the polarisation component with the electric vector perpendicular to the scattering plane and $S_{2}(\theta)$ for the polarisation component with the electric vector parallel to the scattering plane. For unpolarised light, these two scattering components define the phase function of a single particle by

$$
p_{r}(\theta)=\frac{\left|S_{1}(\theta)\right|^{2}+\left|S_{2}(\theta)\right|^{2}}{2|k|^{2} C_{s}},
$$

where $C_{s}$ is the scattering cross section of the particle, $k=2 \pi n_{\text {med }} / \lambda$ is the wave number, and $n_{\text {med }}$ is the refractive index of the host medium. If the host medium is absorbing, $n_{\text {med }}$ is a complex number. The scattering components $\left(S_{1}\right.$ and $\left.S_{2}\right)$ and the scattering cross section of a particle change depending on the radius $r$ of the particle. To indicate this, we let $p_{r}$ denote the phase function of a single particle of radius $r$.

The scattering cross section $C_{s}$ of a particle is the area that would receive the same amount of energy as the particle scatters if we subtend it normal to the incident light. If we multiply the scattering cross section of a particle with the number density of this type of particle in the material, we get the amount of light that will be scattered 
away from a ray of light per unit distance as it propagates through the material, that is, we get the scattering coefficient $\sigma_{s}$. Since the particles may have many sizes with different cross sections and scattering components, the scattering coefficient is an integral over particle radii $r$ :

$$
\sigma_{s}=\int_{r_{\min }}^{r_{\max }} C_{S}(r) N(r) \mathrm{d} r .
$$

The number density in this expression is $N(r) \mathrm{d} r$ while $N(r)$ itself is a number density distribution. It is a distribution because it denotes the number of particles of radius $r$ in the range of particle sizes $\mathrm{d} r$. The justification for this simple combination of scattering cross sections into the macroscopic scattering coefficient is the assumption about independent scattering of the particles.

To keep the phase function normalised, the expression for the macroscopic phase function, often called the ensemble phase function, is:

$$
p(\theta)=\frac{\int_{r_{\text {min }}}^{r_{\text {max }}}\left(\left|S_{1}(\theta)\right|^{2}+\left|S_{2}(\theta)\right|^{2}\right) \mathrm{d} r}{2|k|^{2} \sigma_{s}}=\frac{1}{\sigma_{s}} \int_{r_{\text {min }}}^{r_{\text {max }}} C_{s}(r) p_{r}(\theta) \mathrm{d} r .
$$

As the equation shows, another way to find the ensemble phase function is as a scattering cross section weighted average of the single particle phase functions $p_{r}$ (defined by Equation 4.15).

Now we have an idea about how to find the scattering due to one type of particle in a medium. To deal with several different types of particles, we let $A$ denote the set of homogeneous substances appearing as particles in the host medium. Then $p_{i}, \sigma_{s, i}$, and $\sigma_{t, i}$ denote the phase function and the scattering and extinction coefficients for every individual particle inclusion $i \in A$. Once the phase function has been determined for each individual particle inclusion, the ensemble phase function is computed using a scattering coefficient weighted average:

$$
p(\theta)=\frac{1}{\sigma_{s}} \sum_{i \in A} \sigma_{s, i} p_{i}(\theta) .
$$

Considering the number of single particle phase functions required to approximate the ensemble phase function $p(\theta)$, it is only practical to either tabulate the phase function or to use the ensemble asymmetry parameter $g$ (see below) with one of the standard phase functions, e.g. the Henyey-Greenstein phase function which is defined by [29]

$$
p(\theta)=\frac{1}{4 \pi} \frac{1-g^{2}}{\left(1+g^{2}-2 g \cos \theta\right)^{3 / 2}} .
$$

A more exact option is to use a multi-lobed phase function where the HenyeyGreenstein function replaces $p_{i}(\theta)$ in Equation 4.17. The asymmetry parameter is defined by the integral over all solid angles of the cosine weighted phase function: 


$$
g=\int_{4 \pi} p(\theta) \cos \theta \mathrm{d} \omega
$$

If the asymmetry parameter is computed for single particles and individual particle inclusions, we can compute the ensemble asymmetry parameter as a weighted average using the same weights as for the ensemble phase function.

Because we assume that particles scatter light independently, not only scattering cross sections are additive (see Equation 4.16), but also scattering coefficients (and extinction coefficients) are additive. Finding the bulk scattering coefficient is straightforward:

$$
\sigma_{s}=\sum_{i \in A} \sigma_{s, i}
$$

Note that volume fractions are not included in this formula, because they are a part of the number density distributions.

In a transparent medium, the extinction coefficient is defined by an equivalent sum, but in an absorbing medium an important correction must be made. Since the host medium is a part of the extinction process, a non-absorbing particle will reduce the extinction of the bulk medium. This means that the extinction cross sections can be negative [30]. The extinction cross section resulting from the Lorenz-Mie theory is, in other words, relative to the absorption of the host medium and the necessary correction is to include the host medium absorption $\sigma_{a \text {,med }}$ in the sum. For this purpose, we compute the bulk extinction coefficient for particles in an absorbing medium by

$$
\sigma_{t}=\sigma_{a, \text { med }}+\sum_{i \in A} \sigma_{t, i}
$$

and the bulk absorption coefficient is given by the simple relation $\sigma_{a}=\sigma_{t}-\sigma_{s}$. These bulk coefficients are never negative.

To compute the refractive index of the bulk medium $n_{\text {bulk }}$, we follow van de Hulst's [31] derivation of a formula for the effective index of refraction, but we remove the assumptions of non-absorbing media and particles of only one radius. This gives the following approximate relation for the real part of the bulk refractive index:

$$
\operatorname{Re}\left(n_{\text {bulk }}(\lambda)\right)=\operatorname{Re}\left(n_{\text {med }}(\lambda)\right)+\lambda \sum_{i \in A_{r_{\text {min }}}} \int_{r_{\text {max }}} \operatorname{Im}\left(\frac{S_{i, r, \lambda}(0)}{k^{2}}\right) N_{i}(r) \mathrm{d} r,
$$

where $S_{i, r, \lambda}(0)=S_{1}(0)=S_{2}(0)$ is the amplitude in the forward direction of the wave of wavelength $\lambda$ scattered by a type $i$ particle of radius $r, N_{i}(r)$ is the number density distribution, and $k$ is the wave number. The imaginary part is found by its relation to the bulk absorption coefficient:

$$
\operatorname{Im}\left(n_{\text {bulk }}(\lambda)\right)=\sigma_{a}(\lambda) \frac{\lambda}{4 \pi}
$$




\subsubsection{Lorenz-Mie Theory}

Lorenz [32] and Mie [33] showed that when the scattering components are expanded using spherical functions they are defined, for a homogeneous plane wave, by [32, $33,31,34]$

$$
\begin{aligned}
& S_{1}(\theta)=\sum_{n=1}^{\infty} \frac{2 n+1}{n(n+1)}\left(a_{n} \pi_{n}(\cos \theta)+b_{n} \tau_{n}(\cos \theta)\right) \\
& S_{2}(\theta)=\sum_{n=1}^{\infty} \frac{2 n+1}{n(n+1)}\left(a_{n} \tau_{n}(\cos \theta)+b_{n} \pi_{n}(\cos \theta)\right),
\end{aligned}
$$

where the functions $\pi_{n}$ and $\tau_{n}$ are related to the Legendre polynomials $P_{n}$ as follows:

$$
\begin{aligned}
& \pi_{n}(\cos \theta)=\frac{P_{n}^{1}(\cos \theta)}{\sin \theta}=\frac{\mathrm{d} P_{n}(\cos \theta)}{\mathrm{d}(\cos \theta)} \\
& \tau_{n}(\cos \theta)=\frac{\mathrm{d} P_{n}^{1}(\cos \theta)}{\mathrm{d} \theta}=\cos \theta \pi_{n}(\cos \theta)-\sin ^{2} \theta \frac{\mathrm{d} \pi_{n}(\cos \theta)}{\mathrm{d}(\cos \theta)}
\end{aligned}
$$

Their numeric evaluation can be found in standard references on Lorenz-Mie theory $[35,36]$.

It remains to give expressions for the Lorenz-Mie coefficients $a_{n}$ and $b_{n}$. Suppose the refractive index of the particle is $n_{p}$. Then the coefficients are given, in the far field, by $[32,33,31,34]$

$$
\begin{aligned}
a_{n} & =\frac{n_{\mathrm{med}} \psi_{n}^{\prime}(y) \psi_{n}(x)-n_{p} \psi_{n}(y) \psi_{n}^{\prime}(x)}{n_{\mathrm{med}} \psi_{n}^{\prime}(y) \zeta_{n}(x)-n_{p} \psi_{n}(y) \zeta_{n}^{\prime}(x)} \\
b_{n} & =\frac{n_{p} \psi_{n}^{\prime}(y) \psi_{n}(x)-n_{\mathrm{med}} \psi_{n}(y) \psi_{n}^{\prime}(x)}{n_{p} \psi_{n}^{\prime}(y) \zeta_{n}(x)-n_{\mathrm{med}} \psi_{n}(y) \zeta_{n}^{\prime}(x)},
\end{aligned}
$$

where the primes ' denote derivative. The spherical functions $\psi_{n}(z)$ and $\zeta_{n}(z)$ are known as Riccati-Bessel functions. They are related to the spherical Bessel functions $j_{n}(z)$ and $y_{n}(z)$ as follows:

$$
\begin{aligned}
\psi_{n}(z) & =z j_{n}(z) \\
\zeta_{n}(z) & =z\left(j_{n}(z)-i y_{n}(z)\right) .
\end{aligned}
$$

The argument $z$ is an arbitrary complex number, the arguments $x$ and $y$ used for the Lorenz-Mie coefficients are related to particle and host media as follows:

$$
x=\frac{2 \pi r n_{\mathrm{med}}}{\lambda} \text { and } y=\frac{2 \pi r n_{p}}{\lambda},
$$

where $\lambda$ is the wavelength in vacuum and $r$ is the radius of the spherical particle.

When computers came around, it turned out to be quite difficult to find a numerically stable way of evaluating the spherical functions $\psi_{n}$ and $\zeta_{n}$ for complex argu- 
ments. Eventually, the numerical difficulties were solved for complex $y$ [37, 35, 38]. This is sufficient for the traditional Lorenz-Mie theory with a non-absorbing host medium. When people started considering spheres in an absorbing host, starting with Mundy et al. [39], it became necessary to find a robust way of evaluating the Lorenz-Mie coefficients for complex $x$ as well. This is considerably more difficult. The following describes a robust evaluation scheme proposed by Frisvad et al. [28].

In the case of an absorbing host medium, $n_{\text {med }}$ has an imaginary part and then the parameter $x$ is complex. The consequence is that most numerical evaluation schemes become unstable because the Riccati-Bessel functions enter the exponential domain and run out of bounds.

To avoid the ill-conditioning of the Riccati-Bessel functions $\psi_{n}$ and $\zeta_{n}$, the Lorenz-Mie coefficients (4.21-4.22) are rewritten in a form involving only ratios between them [37]

$$
\begin{aligned}
& a_{n}=\frac{\psi_{n}(x)}{\zeta_{n}(x)} \frac{n_{\mathrm{med}} A_{n}(y)-n_{p} A_{n}(x)}{n_{\mathrm{med}} A_{n}(y)-n_{p} B_{n}(x)} \\
& b_{n}=\frac{\psi_{n}(x)}{\zeta_{n}(x)} \frac{n_{p} A_{n}(y)-n_{\mathrm{med}} A_{n}(x)}{n_{p} A_{n}(y)-n_{\mathrm{med}} B_{n}(x)} .
\end{aligned}
$$

Here $A_{n}(z)$ and $B_{n}(z)$ denote the logarithmic derivatives of $\psi_{n}(z)$ and $\zeta_{n}(z)$ respectively:

$$
A_{n}(z)=\frac{\psi_{n}^{\prime}(z)}{\psi_{n}(z)} \quad \text { and } \quad B_{n}(z)=\frac{\zeta_{n}^{\prime}(z)}{\zeta_{n}(z)} .
$$

The ratio $A_{n}$ is only numerically stable with downward recurrence. Therefore the following formula is employed for its evaluation [37]

$$
A_{n}(z)=\frac{n+1}{z}-\left(\frac{n+1}{z}+A_{n+1}(z)\right)^{-1} .
$$

This formula is also valid for the ratio $B_{n}$, but then it is unfortunately unstable for both upward and downward recurrence [40]. Instead, we use a different formula for $B_{n}$ which has been developed by Mackowski et al. [41] in the field of multilayered particles embedded in a non-absorbing medium. It is numerically stable with upward recurrence for any complex argument [41]:

$$
\begin{aligned}
B_{n}(z) & =A_{n}(z)+\frac{i}{\psi_{n}(z) \zeta_{n}(z)} \\
\psi_{n}(z) \zeta_{n}(z) & =\psi_{n-1}(z) \zeta_{n-1}(z)\left(\frac{n}{z}-A_{n-1}(z)\right)\left(\frac{n}{z}-B_{n-1}(z)\right) .
\end{aligned}
$$

It remains to give a recurrence relation for the ratio $\psi_{n}(z) / \zeta_{n}(z)$ in Equations 4.23 and 4.24. Recent developments in the context of multilayered particles, provide a recurrence relation that works well for $\operatorname{small} \operatorname{Im}(z)[42,43]$ : 


$$
\frac{\psi_{n}(z)}{\zeta_{n}(z)}=\frac{\psi_{n-1}(z)}{\zeta_{n-1}(z)} \frac{B_{n}(z)+n / z}{A_{n}(z)+n / z} .
$$

The restriction to small $\operatorname{Im}(z)$ is not a problem in graphics applications, as a larger $\operatorname{Im}(z)$ means that the host medium is highly absorbing, and then we would not be able to see the effect of particle scattering anyway.

The amplitude functions (4.19-4.20) are defined by an infinite sum, and in order to get a decent approximation, we must find an appropriate number of terms $M$ to sum. This is also necessary for initialisation of the downward recurrence (4.25) which computes $A_{n}(x)$ and $A_{n}(y)$. A formula determining $M$, which has both an empirical $[38,41]$ and a theoretical [40] justification, is

$$
M=\left\lceil|x|+p|x|^{1 / 3}+1\right\rceil
$$

where $p=4.3$ gives a maximum error of $10^{-8}$. It is possible to calculate an approximate initial value for the downward recurrence (4.25), but, as explained by Dave [35], the recurrence is not sensitive to the initial value, and therefore we can arbitrarily choose $A_{M}(z)=0$.

Once $A_{0}(z), \ldots, A_{M}(z)$ have been computed for both $z=x$ and $z=y$, we are able to find the ratios $B_{n}(x)$ and $\psi_{n}(x) / \zeta_{n}(x)$ as well as the Lorenz-Mie coefficients, $a_{n}$ and $b_{n}$, step by step. Note that there is no need to store $B_{n}(x)$ and $\psi_{n}(x) / \zeta_{n}(x)$ since they are computed using upward recurrences (4.26-4.28). These recurrences should be initialised by

$$
\begin{aligned}
B_{0}(z) & =i \\
\psi_{0}(z) \zeta_{0}(z) & =\frac{1}{2}\left(1-e^{i 2 z}\right) \\
\psi_{0}(z) / \zeta_{0}(z) & =\frac{1}{2}\left(1-e^{-i 2 z}\right) .
\end{aligned}
$$

Recall that there is a direct relationship between wavelength $\lambda$ and the size parameters $x$ and $y$. This tells us that the Lorenz-Mie coefficients are spectrally dependent and should preferably be sampled at different wavelengths. They also depend on the particle radius $r$ and are valid for spherical particles of arbitrary size as long as they do not exhibit diffuse reflection (which is only possible if the particle size greatly exceeds the wavelength and, even so, the surface of the particle might still be smooth) [31]. Furthermore, the equations provided in this section reveal that the complex refractive index of each particle inclusion, as well as that of the host medium, are needed as input parameters for computing the optical properties of a scattering material.

This robust way of computing the Lorenz-Mie coefficients enables us to compute the scattering amplitudes $S_{1}$ and $S_{2}$ (using Equations 4.19 and 4.20). With these, we are able to find the extinction and scattering cross sections as well as the phase function of the particle. These are all well defined quantities for particles in a nonabsorbing medium. For a particle in an absorbing medium, the scattering cross section is a problematic quantity because the resulting formula depends on the distance to the observer. 
When particles are embedded in an absorbing host, the extinction cross section $C_{t}$ is the only well defined observable quantity [30]. It is computed using an optical theorem first presented by van de Hulst [44, 31]. The original theorem by van de Hulst is valid for particles of arbitrary shape and size, but it only applies to a nonabsorbing host medium. To account for an absorbing host, we use a slightly modified equation presented by Bohren and Gilra [30]:

$$
C_{t}=4 \pi \operatorname{Re}\left(\frac{S(0)}{k^{2}}\right)
$$

where $S(0)=S_{1}(0)=S_{2}(0)$ is the amplitude in the forward direction of the scattered wave and $k=2 \pi n_{\text {med }} / \lambda$ is the wave number. Since the host medium was assumed by van de Hulst to be non-absorbing, $n_{\text {med }}$ and therefore also $k$ were assumed real and moved outside the Re operator (which takes the real part of a complex number). This is not allowed if the host medium is absorbing as the result would be a meaningless complex extinction coefficient. Correction by discarding the imaginary part of the result would not be a good approximation (except when particle absorption is considerably stronger than that of the host medium [30]). Inserting the expression for $S(0)$ in this optical theorem (4.29), we get

$$
C_{t}=\frac{\lambda^{2}}{2 \pi} \sum_{n=1}^{\infty}(2 n+1) \operatorname{Re}\left(\frac{a_{n}+b_{n}}{n_{\text {med }}^{2}}\right) \text {. }
$$

A form has not been found for the scattering cross section $C_{s}$ which is independent of the distance to the observer, but we still have to approximate $C_{S}$ to evaluate the radiative transfer equation (4.1). We use a far-field approximation which has been reported to be consistent with measured data $[45,46]$. The chosen formula is identical to the scattering cross section for transparent media except for two correction terms: an exponential term and a geometrical term $\gamma$. The formula is

$$
C_{s}=\frac{\lambda^{2} e^{-4 \pi r \operatorname{Im}\left(n_{\mathrm{med}}\right) / \lambda}}{2 \pi \gamma\left|n_{\mathrm{med}}\right|^{2}} \sum_{n=1}^{\infty}(2 n+1)\left(\left|a_{n}\right|^{2}+\left|b_{n}\right|^{2}\right),
$$

where $r$ in the exponential term is the uncertain part of the equation because it ought to be the distance to where the scattered wave is observed. This distance is unknown, and consequently it has been projected to the particle surface, such that $r$ denotes the particle radius.

The geometrical term $\gamma$ accounts for the fact that the incident wave changes over the surface of the particle as a consequence of the absorbing host medium. It is defined by [39]

$$
\gamma=\frac{2\left(1+(\alpha-1) e^{\alpha}\right)}{\alpha^{2}}
$$

where $\alpha=4 \pi r \operatorname{Im}\left(n_{\text {med }}\right) / \lambda$ and $\gamma \rightarrow 1$ for $\alpha \rightarrow 0$. Note that $\alpha$ is 0 when the medium is transparent and close to 0 for small particles in a weakly absorbing medium. To avoid numerical errors, one should use $\gamma=1$ for $\alpha<10^{-6}$. 
The precision of the far field approximation $(4.30,4.31)$ has recently been reviewed [47] and compared to experimental data $[45,46]$. The conclusion is that it (as expected) does not give entirely accurate results, but it does give physically plausible results. It is also concluded that significant errors can result if the absorption of the host medium is ignored (this is especially true when the size parameter $x$ is large).

In the same way that it is possible to formulate expressions for the scattering and extinction cross sections of a spherical particle using Lorenz-Mie theory, it is also possible to derive the following formula for the asymmetry parameter of a single spherical particle [31]:

$$
g=\frac{\sum_{n=1}^{\infty}\left\{\frac{n(n+2)}{n+1} \operatorname{Re}\left(a_{n} a_{n+1}^{*}+b_{n} b_{n+1}^{*}\right)+\frac{2 n+1}{n(n+1)} \operatorname{Re}\left(a_{n} b_{n}^{*}\right)\right\}}{\frac{1}{2} \sum_{n=1}^{\infty}(2 n+1)\left(\left|a_{n}\right|^{2}+\left|b_{n}\right|^{2}\right)},
$$

where the asterisks * denote the complex conjugate.

This concludes the robust scheme for computing the scattering properties of a sphere in a host medium. When using the Lorenz-Mie theory to compute macroscopic scattering properties of a medium (as described in Section 4.3.1), some information about the particle shapes and sizes is needed. We will look at this in Sections 4.3.3 and 4.3.4.

\subsubsection{Number Density Distributions}

Particle size distribution is the common term for distributions that we can use to find the number densities of particles of different sizes. One type of size distribution, which is often encountered in the literature, is the volume frequency distribution $r^{3} N(r)$. Such distributions typically follow a log-normal distribution. Log-normal distributions are often described by a mean particle size $\mu$ and a coefficient of variation $c_{v}=\sigma / \mu$, where $\sigma$ is the standard deviation.

If we find that the volume frequency of some type of particle in a medium follows the log-normal distribution with mean value $\mu$ and standard deviation $\sigma$, the volume frequency distribution is given by

$$
r^{3} N(r)=\frac{1}{r \beta \sqrt{2 \pi}} e^{-\frac{1}{2}\left(\frac{\ln r-\alpha}{\beta}\right)^{2}},
$$

where $r$ is the particle radius and

$$
\alpha=\ln \mu-\frac{1}{2} \ln \left(\frac{\sigma^{2}}{\mu^{2}}+1\right) \quad \text { and } \quad \beta=\sqrt{\ln \left(\frac{\sigma^{2}}{\mu^{2}}+1\right)} .
$$

This type of distribution is commonly observed for small particles. Distributions of larger particles tend to follow a power law. If the particle number density of some 
type of particle in a medium follows a power law, the distribution is given by

$$
N(r)=N_{*} r^{-\alpha}
$$

where $\alpha$ is usually determined empirically and $N_{*}$ is a constant which is determined by the relationship between number density and the volume fraction of the medium occupied by the considered type of particle (Equation 4.33).

Measured data are sometimes available which specify the volume fraction $v_{i}$, $i \in A$, of each particle inclusion that is present in the bulk medium. In any case, volume fractions are a reasonable choice of input parameters. The number density distribution $N(r)$ specifies the number of particles per unit volume with radii in the interval $[r, r+\mathrm{d} r]$. This means that the volume fraction occupied by a particle inclusion, which consists of spherical particles, is

$$
v=\frac{4 \pi}{3} \int_{r_{\min }}^{r_{\max }} r^{3} N(r) \mathrm{d} r .
$$

Suppose we measure the particle size distributions for some sample of material. Then we would have empirical functions or tabulated data that fit the volume fractions of the particles in the original sample. Most probably the original volume fractions are not the volume fractions we desire in our medium. Equation 4.33 is important because it explains how we find the original volume fraction $v_{\text {original }, i}$ of particle type $i$. If the volume fraction $v_{i}$ is desired rather than $v_{\text {original }, i}$, the measured number density distribution should be scaled by $v_{i} / v_{\text {original }, i}$.

\subsubsection{Non-Spherical Particles}

Particles are not always spherical. There exist a number of theories for the scattering of other perfect mathematical shapes like cylinders, hexagonal columns and plates, etc. They are useful because some materials actually do consist of particles approximately of these shapes. Halos are, for example, the result of scattering by hexagonal ice crystals in the atmosphere. Instead of the mathematical approach, let us use a more practical approach for non-spherical particles.

With the theory we have already developed, we are able to approximate nonspherical particles by an appropriate collection of spherical particles. It is not obvious what set of spheres we should choose to model a non-spherical particle in the best way. Many different concepts have been tried: equal-volume spheres, equal-area spheres, etc. The best approach we are aware of is that of Grenfell and Warren [48]. They use volume-to-area equivalent spheres. As opposed to equal-volume and equalarea spheres, the volume-to-area equivalent spheres have proven to be quite exact. They have been tested for cylinders [48], hexagonal columns and plates [49], and hollow columns and plates [50]. In most cases the error is less than 5\%. At least this 
is true for scattering and extinction coefficients. The approximation is, as could be expected, less accurate with respect to the phase function.

To represent a particle of volume $V$ and surface area $A$ by a collection of spheres, the radius of the equivalent spheres is found simply using the volume to surface area ratio of a sphere [48]:

$$
r_{\mathrm{eq}}=3 \frac{\mathrm{V}}{\mathrm{A}} .
$$

Since the number of equivalent spheres is not equal to the number of non-spherical particles, the number density must be adjusted accordingly [48]:

$$
\frac{N_{\mathrm{eq}}}{N}=\frac{3 V}{4 \pi r_{\mathrm{eq}}^{3}} .
$$

The equivalent radius $r_{\mathrm{eq}}$ and the equivalent number density $N_{\mathrm{eq}}$ are then used for computing the optical properties of the material with Lorenz-Mie theory for computing the cross sections of the equivalent spheres. This is a simple and practical approach which gives rather good results. It has been used with the theory presented here to develop an appearance model for natural ice which contains both cylindrically and spheroidally shaped particles [28, 51]. In the milk case study (Section 4.4), the volume-to-area equivalent size distribution is reported directly in the literature, so the radius and number density adjustments are not necessary.

\subsubsection{Scattering of a Gaussian Beam}

In wave optics, a generalised version of the Lorenz-Mie theory $[52,53]$ would be needed to model the scattering by a particle of a shaped beam such as laser. However, we are using the scattering by particles in a geometrical optics context. The scattering of a ray, which traces an infinitely thin part of the wavefront, is adequately modelled by the scattering of a plane wave. Thus we do not employ the generalised Lorenz-Mie theory, but model a Gaussian beam (a laser source) as described in Section 4.2.3.

When we render an image using the algorithm described in Section 4.2.5, the laser source poses a problem. Rays are traced from the observer and new directions are sampled at every scattering event. Since the laser source is small and collimated, the chance of a ray hitting the laser source from the right direction is almost nonexisting. To solve this problem, we use bidirectional path tracing [54]. As described in Section 4.2, rays are traced from the observer through each pixel in the image into the scene. To account for a laser source, we also sample a position on the laser source and trace a ray from this position to the first scattering event in a medium. For every ray from the observer that reaches this medium, we compute the contribution from the scattered laser light. Every time all pixels have been sampled once by rays from the observer, a new sample ray is traced from the laser source. This is how we accommodate a laser source in the path tracing algorithm. In the following, we will use the theory to predict the appearance of milk. 
Table 4.1 The coefficients for the empirical formula (4.34) by Quan and Fry [57].

\begin{tabular}{lll}
\hline$n_{1}=1.779 \cdot 10^{-4}$ & $n_{4}=-2.02 \cdot 10^{-6}$ & $n_{7}=-0.00423$ \\
$n_{2}=-1.05 \cdot 10^{-6}$ & $n_{5}=15.868$ & $n_{8}=-4382$ \\
$n_{3}=1.6 \cdot 10^{-8}$ & $n_{6}=0.01155$ & $n_{9}=1.1455 \cdot 10^{6}$ \\
\hline
\end{tabular}

\subsection{Milk as a Case Study}

Milk consists roughly of an emulsion of milkfat globules; a colloidal suspension of protein particles; and lactose, soluble proteins, minerals, vitamins, acids, enzymes, and other components dissolved in water [55]. About $80 \%$ of the protein in milk is casein protein. Most of this casein, about 95\% [56], exists in colloidal particles known as casein micelles. From an optical point of view, milk can then be treated as two different types of nearly spherical particles, namely fat globules and casein micelles, suspended in a host medium with almost the same optical properties as pure water. The absorption spectrum of the host medium needs to be adjusted because of dissolved vitamin B2 (riboflavin) which exhibits absorption in the visible range of the spectrum. In fact riboflavin is also fluorescent, but we will not take that into account. What we use is, in other words, a simplified model of milk, but it should be sufficient for considering the appearance of milk.

\subsubsection{Particle Composition}

The host medium is water in which many different components are dissolved. For the real part of the refractive index of the milk host, we use the refractive index for pure fresh water $(S=0 \%$ in Equation 4.34).

Quan and Fry [57] have developed an empirical formula for computing the real part of the refractive index of pure water or brine as a function of salinity $S$, temperature $T$, and wavelength $\lambda$. It is as follows [57]:

$$
\begin{aligned}
n_{\text {water }}^{\prime}(\lambda, T, S)= & 1.31405+\left(n_{1}+n_{2} T+n_{3} T^{2}\right) S+n_{4} T^{2} \\
& +\frac{n_{5}+n_{6} S+n_{7} T}{\lambda}+\frac{n_{8}}{\lambda^{2}}+\frac{n_{9}}{\lambda^{3}}
\end{aligned}
$$

The coefficients are listed in Table 4.1. This formula describes the dependency of $n_{\text {water }}^{\prime}$ on salinity in the range $0 \%$ o $<<35 \%$, temperature in the range $0{ }^{\circ} \mathrm{C}<T<$ $30^{\circ} \mathrm{C}$, and wavelength in the range $400 \mathrm{~nm}<\lambda<700 \mathrm{~nm}$. Moreover Huibers [58] has reported that the same formula is valid over a broader spectrum of wavelengths ( $200 \mathrm{~nm}<\lambda<1100 \mathrm{~nm}$ ) than originally assumed.

To find the imaginary part of the refractive index of the milk host, we make a correction for the imaginary part of the refractive index of pure water. The dissolved component exhibiting the most significant absorption in the visible range is vitamin B2 (riboflavin). Spectral data for the absorption of vitamin B2 are available in 
Table 4.2 Imaginary part of the refractive index for pure water [61], riboflavin [59] $(0.17 \mathrm{mg}$ pr. $100 \mathrm{~g}$ solution), milk host $n_{\text {milk }}^{\prime \prime}$, and milk fat $n_{\text {fat }}^{\prime \prime}$.

\begin{tabular}{rllll}
\hline$\lambda[\mathrm{nm}]$ & $n_{\text {water }}^{\prime \prime}$ & $n_{\text {riboflavin }}^{\prime \prime}$ & $n_{\text {milk }}^{\prime \prime}$ & $n_{\text {fat }}^{\prime \prime}$ \\
\hline 375 & $3.393 \cdot 10^{-10}$ & $2.927 \cdot 10^{-7}$ & $2.93 \cdot 10^{-7}$ & $4.0 \cdot 10^{-6}$ \\
400 & $2.110 \cdot 10^{-10}$ & $2.603 \cdot 10^{-7}$ & $2.60 \cdot 10^{-7}$ & $6.4 \cdot 10^{-6}$ \\
25 & 1.617 & 3.363 & 3.36 & 8.6 \\
50 & 3.302 & 4.096 & 4.10 & $1.1 \cdot 10^{-5}$ \\
75 & 4.309 & 3.323 & 3.33 & 1.1 \\
500 & $8.117 \cdot 10^{-10}$ & $1.076 \cdot 10^{-7}$ & $1.08 \cdot 10^{-7}$ & $1.0 \cdot 10^{-5}$ \\
25 & $1.742 \cdot 10^{-9}$ & $5.470 \cdot 10^{-8}$ & $5.64 \cdot 10^{-8}$ & $4.7 \cdot 10^{-6}$ \\
50 & 2.473 & 5.772 & 6.02 & 4.6 \\
75 & 3.532 & 7.554 & 7.91 & 4.7 \\
600 & $1.062 \cdot 10^{-8}$ & $6.889 \cdot 10^{-8}$ & $7.95 \cdot 10^{-8}$ & $4.9 \cdot 10^{-6}$ \\
25 & 1.410 & 7.169 & 8.58 & 5.0 \\
50 & 1.759 & 7.563 & 9.32 & 5.0 \\
75 & 2.406 & 4.967 & 7.37 & 5.1 \\
700 & $3.476 \cdot 10^{-8}$ & $7.937 \cdot 10^{-8}$ & $1.14 \cdot 10^{-7}$ & $5.2 \cdot 10^{-6}$ \\
25 & 8.591 & 4.683 & 1.33 & 5.2 \\
50 & $1.474 \cdot 10^{-7}$ & 7.287 & 2.20 & 5.2 \\
775 & $1.486 \cdot 10^{-7}$ & $8.626 \cdot 10^{-8}$ & $2.35 \cdot 10^{-7}$ & $5.2 \cdot 10^{-6}$ \\
\hline
\end{tabular}

The milk host spectrum is $n_{\text {water }}^{\prime \prime}+n_{\text {riboflavin }}^{\prime \prime}$ The milk fat spectrum is from Michalski et al. [62].

the PhotochemCAD application ${ }^{1}$ [59]. The absorption coefficient is not measured directly, instead the absorbance $D$ is measured. The absorption coefficient is calculated from the absorbance using a molar absorption coefficient $\varepsilon$ for some wavelength. A molar absorption coefficient of riboflavin is $\varepsilon(266.5 \mathrm{~nm})=3.3 \cdot 10^{6} \mathrm{M}^{-1} \mathrm{~m}^{-1}$ [60]. The following formula finds the remaining molar absorption coefficients for riboflavin using the absorbance data:

$$
\varepsilon(\lambda)=\frac{\varepsilon(266.5 \mathrm{~nm})}{D(266.5 \mathrm{~nm})} \ln (10) D(\lambda)
$$

The natural content in milk of riboflavin is $0.17 \mathrm{mg}$ per $100 \mathrm{~g}$ milk [56]. Using the molar mass of riboflavin which is $376.3682 \mathrm{~g} / \mathrm{mol}$, we find that the natural concentration of riboflavin in milk is

$$
c=\frac{0.17 \mathrm{mg}}{376.3682 \mathrm{~g} / \mathrm{mol}} / \frac{100 \mathrm{~g}}{1.03 \mathrm{~g} / \mathrm{mL}}=4.65 \cdot 10^{-6} \mathrm{~mol} / \mathrm{L} .
$$

By multiplication of the molar absorption coefficient with this concentration, we obtain the absorption coefficient of riboflavin which is converted to the imaginary part of a refractive index (Equation 4.18) and added to the imaginary part of the refractive index for pure water to obtain the imaginary part for the milk host, $n_{\text {milk }}^{\prime \prime}$. See Table 4.2.

\footnotetext{
${ }^{1}$ http://omlc.ogi.edu/spectra/PhotochemCAD/abs_html/riboflavin.html
} 


\section{The Fat Inclusion}

Walstra and Jenness [63] have found experimentally that the real part of the refractive index of milk fat approximately follows the function

$$
n_{\text {fat }}^{\prime}(\lambda, T)=\sqrt{\frac{(b(T)+2) \lambda^{2}-0.03}{(b(T)-1) \lambda^{2}-0.03}},
$$

where wavelength is measured in $\mu \mathrm{m}$ and $b$ is found using a measurement of the refractive index at the temperature $T$. We use measurements by Michalski et al. [62] since they also present the wavelength dependent imaginary part of the refractive index. They find $n_{\text {fat }}^{\prime}\left(0.589 \mu \mathrm{m}, 20^{\circ} \mathrm{C}\right)=1.461$ which gives $b\left(20^{\circ} \mathrm{C}\right)=3.73$. This corresponds well to the $b\left(40^{\circ} \mathrm{C}\right)=3.77$ reported by Walstra and Jenness [63]. Table 4.2 includes the imaginary part of the refractive index for milk fat $n_{\text {fat }}^{\prime \prime}$ as we read it from the curve reported by Michalski et al. [62].

The volume frequency of the fat globules follows a log-normal distribution [64] (cf. Equation 4.32). The mean of the volume-to-area equivalent sphere radii $r_{\text {eq,fat }}$ of the fat globules change depending on the volume fraction of the globules in the milk. By a least-squares, two-piece fit to measured data reported by Olson et al. [65], we have found a functional expression describing this relationship:

$$
r_{43, \mathrm{fat}}=\left\{\begin{array}{cl}
-0.2528 w_{f}^{2}+1.419 w_{f} & \text { for } w_{f}<2.0 \\
1.456 w_{f}^{0.36} & \text { otherwise }
\end{array}\right.
$$

where $r_{43 \text {,fat }}$ is measured in $\mu \mathrm{m}$. The relationship between $r_{43, \text { fat }}$ and $r_{\mathrm{eq} \text {,fat }}$ is [64]

$$
r_{\mathrm{eq}, \mathrm{fat}}=r_{43, \mathrm{fat}} /\left(c_{v, \text { fat }}^{2}+1\right) .
$$

The radius $r_{43 \text {,fat }}$ is used since it can be estimated empirically with good accuracy [64]. The coefficient of variation $c_{v, \text { fat }}$ is usually between 0.4 and 1.2 in normal milk. Reasonable limits for the range of fat globule radii are $r_{\text {min,fat }}=0.005 \mu \mathrm{m}$ and $r_{\text {max }, \text { fat }}=10 \mu \mathrm{m}$.

\section{The Protein Inclusion}

The refractive index of casein micelles is not readily available in the literature. For comparison to goat's milk it has been determined to be the following for cow's milk [66]:

$$
n_{\text {casein }}=1.503 \text {. }
$$

This value is assumed to be constant in the visible range and absorption of the casein micelles is neglected.

Structure and size distribution of casein micelles is still being disputed in the literature. Recent research on the matter is discussed by Gebhardt et al. [67]. Most investigations are based on either light scattering or electron microscopy. Light scat- 
Table 4.3 A summary of the microscopic properties of cow's milk.

\begin{tabular}{llll}
\hline Property & Milk host & Fat globules & Casein micelles \\
\hline$n^{\prime}$ & Equation 4.34 & Equation 4.35 & 1.503 \\
$n^{\prime \prime}$ & Table 4.2 & Table 4.2 & 0.00 \\
$r$ & & {$[0.005 \mu \mathrm{m}, 10 \mu \mathrm{m}]$} & {$[0 \mathrm{~nm}, 150 \mathrm{~nm}]$} \\
$N(r)$ & & Equation 4.32 & Equation 4.32 \\
\hline
\end{tabular}

Table 4.4 Densities for computing of milk fat and casein volume fractions using weight percents.

\begin{tabular}{lll}
\hline$\rho_{\text {fat }}$ & $\rho_{\text {protein }}$ & $\rho_{\text {milk }}$ \\
\hline $0.915 \mathrm{~g} / \mathrm{mL}$ & $1.11 \mathrm{~g} / \mathrm{mL}$ & $1.03 \mathrm{~g} / \mathrm{mL}$ \\
\hline
\end{tabular}

Measured by Walstra and Jenness [63] at $20^{\circ} \mathrm{C}$.

tering approaches find micelles of large average size while electron microscopy report a large number of very small casein particles in addition to the larger micelles. Sometimes these very small particles are excluded from the reported size distribution since they are regarded to represent non-micellar casein or single sub-micelles. No matter what we call these very small particles, they scatter light as do the larger aggregates and therefore should be included in the size distribution employed for the Lorenz-Mie calculations.

A size distribution based on electron microscopy, which includes the single submicelles in the distribution, was reported by Schmidt et al. [68]. They found the mean $r_{\text {eq,casein }}=43 \mathrm{~nm}$ and showed that a $\log$-normal distribution (4.32) of $r /\left(r_{\text {max,casein }}-\right.$ $r)$ is a good fit of the measured volume frequency distribution. The limits for the casein micelle radii are $r_{\text {min,casein }}=0 \mathrm{~nm}$ and $r_{\text {max,casein }}=150 \mathrm{~nm}$.

The microscopic properties of milk are summarised in Table 4.3.

\subsubsection{Appearance Model}

To model the concentration of fat and protein we use wt.- $\%$ ( $\mathrm{g}$ per $100 \mathrm{~g}$ milk), since this value is used on content declarations on the side of milk cartons. In the remainder of this chapter we let $w_{f}$ and $w_{p}$ denote the wt.- $\%$ of fat and protein respectively. To translate wt.- $\%$ into volume fractions, we use the densities given by Walstra and Jenness [63]. They are summarised in Table 4.4. Casein micelles make up about $80 \% \cdot 95 \%=76 \%$ of the protein volume fraction (see above). This means that

$$
v_{\text {fat }}=\frac{w_{f} / \rho_{\text {fat }}}{100 \mathrm{~g} / \rho_{\text {milk }}} \quad \text { and } \quad v_{\text {casein }}=0.76 \frac{w_{p} / \rho_{\text {protein }}}{100 \mathrm{~g} / \rho_{\text {milk }}}
$$



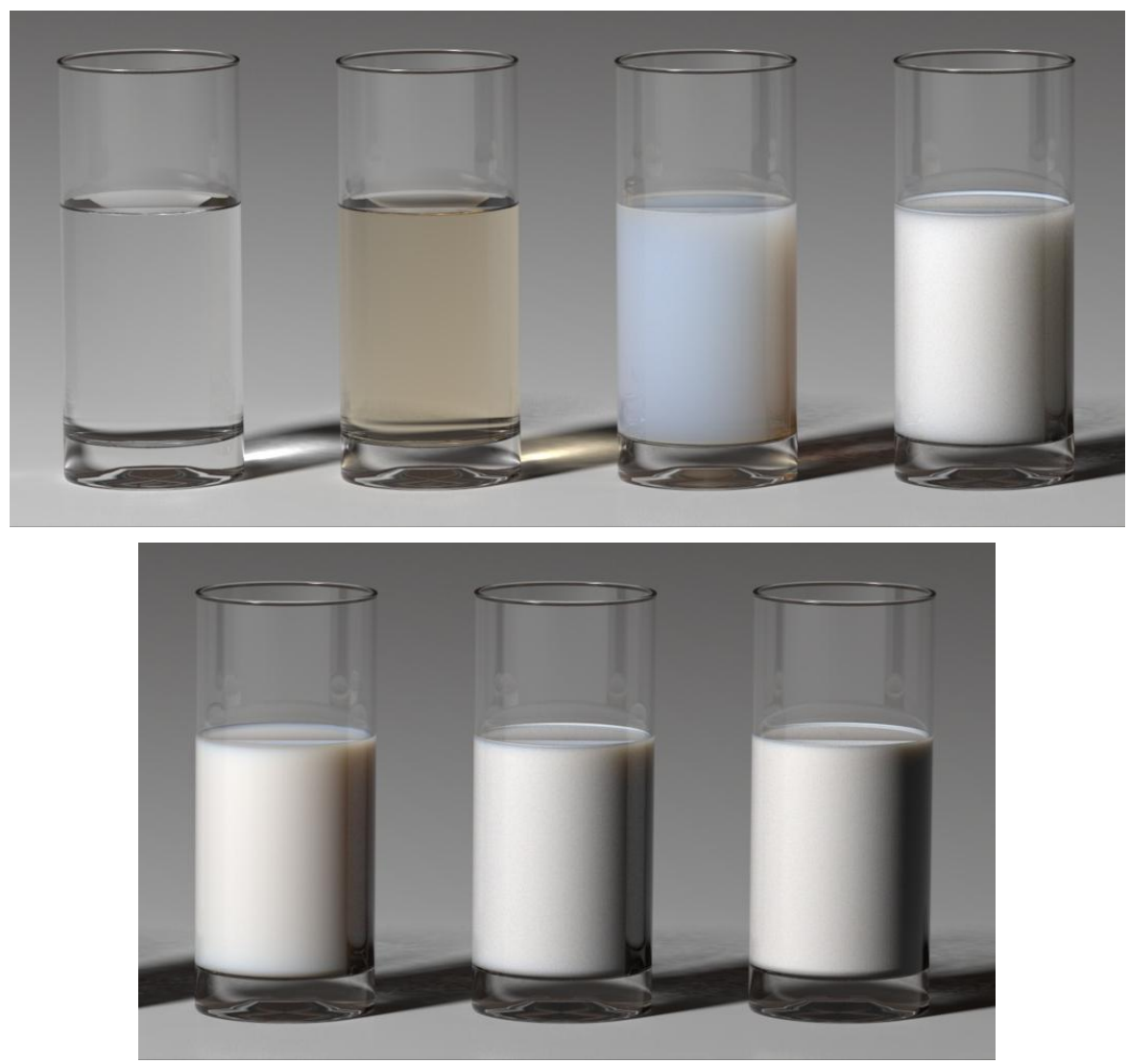

Fig. 4.1 Rendered images of the components in milk (top row) as well as mixed concentrations (bottom row). From top left to bottom right the glasses contain: pure water, water and vitamin B2, water and protein, water and fat, skimmed milk, regular milk, and whole milk.

This simple translation from fat and protein contents to volume fractions of the particle inclusions in the milk means that we have an appearance model with the following parameters:

- Fat content $w_{f}$

- Protein content $w_{p}$.

These two parameters are all we need to model most types of milk.

\subsubsection{Results}

The protein content of the milk we buy in a grocery store is usually around $w_{p}=$ 3.4 wt.- $\%$ while the content of fat is what we use to distinguish between different 
Fig. 4.2 A simple experimental setup where we can take a picture of a laser pointer shining light into a cup of milk.

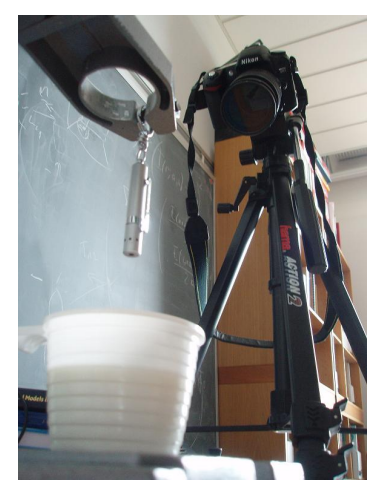

milk products: skimmed milk, $w_{f}=0.1 \mathrm{wt} .-\%$; low fat milk, $w_{f}=1.5 \mathrm{wt} .-\%$; whole milk $w_{f}=3.5$ wt. $\%$. This information, and the appearance model described above, enables us to visualise different types of milk. We are also able to show the visual significance of each component in the milk. This is a particular strength of the approach that we take. Knowing the visual significance of the different ingredients in a material is important if we would like to design the appearance of the material or if we would like to interpret the appearance of the material. Figure 4.1 shows the visual significance of different components in milk as well as the predicted appearance of different milk products. Starting leftmost in the top row, the first glass contains pure water, the second includes the absorption of vitamin B2 and is the host medium of the particles in the milk, the third glass contains casein micelles in water, the fourth contains fat globules in water, and the three glasses in the bottom row contain skimmed milk, low fat milk, and whole milk, respectively. The contents of these glasses have all been rendered using the appearance model described in this section. Spectral optical properties (sampled at every $25 \mathrm{~nm}$ ) were computed as described in Section 4.3 for all the milk materials. For rendering, we computed RGB representations of the optical properties by weighted averages using the RGB colour matching functions of a standard human observer [69]. We used homogeneous scattering properties for the milk and the approximative Henyey-Greenstein phase function with an ensemble asymmetry parameter.

The results provided in Figure 4.1 (bottom row) compare only qualitatively to pictures of real milk. Our eyes are the instruments in such a comparison. To find out if our model correctly predicts the appearance of real milk, some sort of quantitative comparison is necessary. We can do a quantitative comparison by constructing a simple experimental setup which we can easily model and render. The setup we choose is photographed in Figure 4.2. It consists of a digital camera on a tripod pointing at the surface of milk in a cup, and some arbitrary device for hanging up a laser pointer over the cup. The experiment is to take a picture of the milk while the laser pointer shines light into it directly from above. The room should be darkened as much as possible, but it is usually not possible nor practical to black it out completely. For this reason, a picture was first taken with the laser pointer turned off and one was then taken with the laser pointer turned on. Subtracting the first picture from 
Laser in skimmed milk - photo

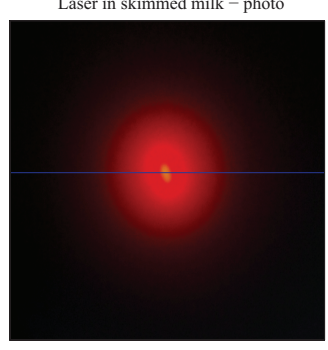

Laser in skimmed milk - computed

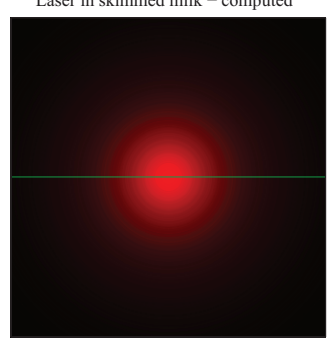

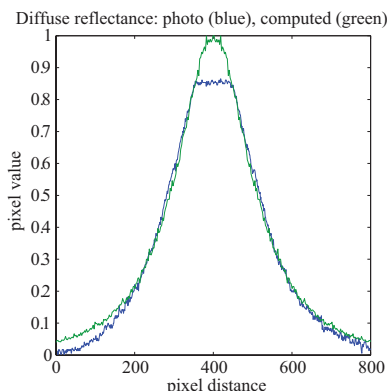

Fig. 4.3 Photographed laser in skimmed milk $\left(w_{f}=0.1 \mathrm{wt} .-\%, w_{p}=3.4 \mathrm{wt} .-\%\right)$, simulated laser in skimmed milk, and comparison of the diffusion curves, that is, the middle lines through the images.

the second removes the background illumination to reveal the scattering of the laser in the milk alone. To avoid scattering of light in the cup itself, the white cup in Figure 4.2 was replaced by a black, opaque cup when the pictures were taken. In addition, the digital camera was and should be configured to process the picture as little as possible.

To model the experimental setup digitally, all we need is a correctly sized cylinder of milk, a laser source placed directly above it, and a camera placed at the right distance from the milk surface. The laser pointer we used had a diameter of $3 \mathrm{~mm}$, power of $\Phi=1 \mathrm{~mW}$, and wavelength of $\lambda=650 \mathrm{~nm}$. This means that all the laser light should go into the red colour band. The horizontal line in the image through the laser spot centre is referred to as the diffuse reflectance curve. We only use the diffuse reflectance curve of the red colour band in our quantitative comparison. This line is perpendicular to the direction toward the camera, so only the distance to the camera is important not the angle. For this reason, we place both camera and laser pointer directly above the milk in our digital scene. To save computations, we only render pixels from the laser spot centre to the right border of the film (half the diffuse reflectance curve), and then we revolve this result around the centre to get a full synthesized image of the laser spot.

The photographed milk, the rendered milk, and the quantitative comparison are provided in Figure 4.3. There are a few differences between theory and experiment that we should discuss. The real camera is overexposed by the reflected laser light while the synthetic camera is not (the top of the blue curve has been cut off). The reason is the background illumination which was subtracted. In this experiment, the background illumination was just enough to overexpose the laser spot in the photograph. Another difference is that the photographed laser spot is not symmetric. It is more elliptically shaped. The reason for this deviation in the rendered image is that the laser source was modelled as a circular disc (see Sec. 4.2.3), and this is not the shape of the light emitted by a standard laser pointer. Finally, the central part of the photographed laser spot is not only red. The most likely explanation is that light penetrates the colour filters in the CCD chip when it is overexposed. This means that some of the red light spills into the other colour bands in the overexposed area of the 
Laser in whole milk - photo

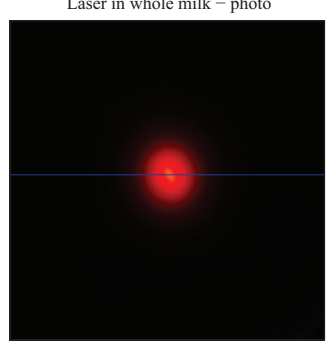

Laser in whole milk - computed

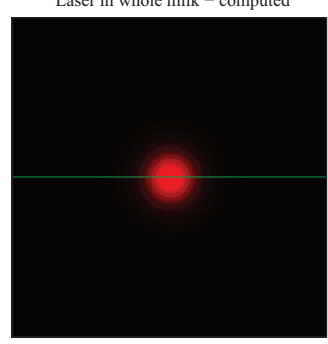

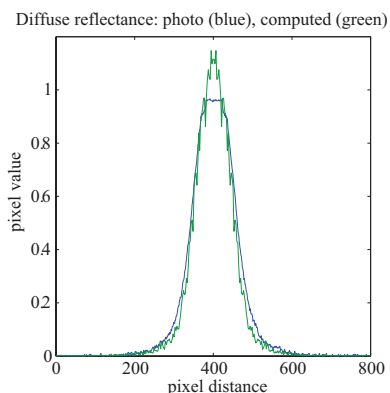

Fig. 4.4 Photographed laser in whole milk $\left(w_{f}=3.5 \mathrm{wt} .-\%, w_{p}=3.4 \mathrm{wt} .-\%\right)$, simulated laser in whole milk, and comparison of the diffusion curves, that is, the middle lines through the images.

image. Apart from these explainable differences, there is surprisingly good quantitative agreement between theory and experiment (the diffuse reflectance curves are close to each other).

From a qualitative perspective, the rotational symmetry of the simulated image is a giveaway which makes it easy for the human eye to spot the synthetic image in comparison to the real one. This might not be as easy if we had not revolved a single line in the image around the centre to save computations. Although it is not physically accurate, the red light spilling into the other colour bands where the light is intense in the photograph convinces the eye that the photograph is more real. This is because the human eye is able to perceive a more intense red colour than what the camera can capture (and what a standard computer screen can display). The added intensity from the other colour bands therefore seems more realistic than a flat red colour. As a final example, to illustrate that the appearance model is reliable for different types of milk, Figure 4.4 compares photographed and simulated laser in whole milk.

\subsection{Conclusion}

The milk example demonstrates that we are able to show the visual significance of the different components in a material by computing synthetic images. It also demonstrates that we are able to predict the appearance for various ratios between the contents. This makes a number of interesting applications possible:

- If you want to design materials with a specific appearance, the appearance model can help you choose the right components to obtain the desired appearance.

- If you want to detect whether a component is present in a material or not, the appearance model can help you visualise the material as it would look with and without the component. 
In other words, synthesised images with a connection to the contents and the physical conditions of a material enable us to learn a lot about the reasons for the appearance of materials.

Considering the input parameters that brought us to the simulated diffuse reflectance curves in Figures 4.3 and 4.4, the presented method is definitely useful for analysis by synthesis. If we simulate a large number of diffuse reflectance curves for milks with different particle inclusions, we can construct numerical methods for analysing the properties of these inclusions from photographed diffuse reflectance curves. The slope of the diffuse reflectance curve, for example, reveals a lot about the fat content of the milk (as is obvious from the figures). Further investigation can lead to more precise models for determining the fat content of milk from a digital photograph.

The Lorenz-Mie theory provides the link from the particle composition of a material to the macroscopic scattering properties that we can use with radiative transfer theory to compute a realistic image. In this way, a century after its formulation, the Lorenz-Mie theory is the key component when we want to predict the appearance of materials using a physical model.

\section{References}

1. R.A. Hall, D.P. Greenberg, A testbed for realistic image synthesis, IEEE Computer Graphics and Applications 3(8), 10 (1983)

2. A.S. Glassner, Principles of Digital Image Synthesis (Morgan Kaufmann Publishers, Inc., San Francisco, California, 1995). A two-volume set.

3. S. Chandrasekhar, Radiative Transfer (Oxford, Clarendon Press, 1950). Unabridged and slightly revised version published by Dover Publications, Inc., in 1960

4. R. Siegel, J.R. Howell, Thermal Radiation Heat Transfer, 4th edn. (Taylor \& Francis, New York, 2002)

5. M. Pharr, G. Humphreys, Physically Based Rendering: From Theory to Implementation (Morgan Kaufmann Publishers, an imprint of Elsevier Inc., 2004)

6. B.T. Phong, Illumination for computer-generated pictures, Communications of the ACM 18(6), $311(1975)$

7. R.V. Klassen, Modeling the effect of the atmosphere on light, ACM Transactions on Graphics 6(3), 215 (1987)

8. D. Jackèl, B. Walter, Modeling and rendering of the atmosphere using Mie-scattering, Computer Graphics Forum 16(4), 201 (1997)

9. A.J. Preetham, P. Shirley, B. Smits, in Proceedings of ACM SIGGRAPH 1999 (ACM Press, 1999), pp. 91-100

10. L.V. Wang, H.I. Wu, Biomedical Optics: Principles and Imaging (John Wiley \& Sons, Inc., Hoboken, New Jersey, 2007)

11. A. Sommerfeld, J. Runge, Anwendung der Vektorrechnung auf die Grundlagen der Geometrischen Optik, Annalen der Physik 340, 277 (1911)

12. P.d. Fermat, Euvres de Fermat: Publiées par les soins de MM. Paul Tannery et Charles Henry sous les auspices du Ministère de l'instruction publique, vol. 2 (Gauthier-Villars et fils, 18911912). Correspondance

13. A. Glassner, Space subdivision for fast ray tracing, IEEE Computer Graphics and Applications 4(10), 15 (1984) 
14. F.W. Jansen, in Data Structures for Raster Graphics: Proceedings of a Workshop Held at Steensel, the Netherlands, from 24-28 June 1985 (Springer, 1986), Eurographics seminars, pp. $57-73$

15. A. Fujimoto, T. Tanaka, K. Iwata, ARTS: Accelerated ray-tracing system, IEEE Computer Graphics and Applications 6(4), 16 (1986)

16. K. Sung, P. Shirley, in Graphics Gems III, ed. by D. Kirk (Academic Press, 1992), pp. 271-274

17. T. Möller, B. Trumbore, Fast, minimum storage ray-triangle intersection, Journal of Graphics Tools 2(1), 21 (1997)

18. A. Fresnel, Mémoire sur la loi des modifications que la réflexion imprime a la lumière polarisée, Mémoires de l'Académie des sciences de l'Institut de France 11, 393 (1832). Presented 7 January 1823

19. T. Whitted, An improved illumination model for shaded display, Communications of the ACM 23(6), 343 (1980). Presented at SIGGRAPH 79

20. R.W. Preisendorfer, Radiative Transfer on Discrete Spaces (Pergamon Press, 1965)

21. A. Ishimaru, Wave Propagation and Scattering in Random Media (Academic Press, New York, 1978). Reissued by IEEE Press and Oxford University Press 1997

22. J.T. Kajiya, B.P. Von Herzen, Ray tracing volume densities, Computer Graphics (Proceedings of ACM SIGGRAPH 84) 18(3), 165 (1984)

23. S.N. Pattanaik, S.P. Mudur, Computation of global illumination in a participating medium by Monte Carlo simulation, The Journal of Visualization and Computer Animation 4(3), 133 (1993)

24. T. Nishita, Y. Miyawaki, E. Nakamae, A shading model for atmospheric scattering considering luminous intensity distribution of light sources, Computer Graphics (Proceedings of ACM SIGGRAPH 87) 21(4), 303 (1987)

25. H. Rushmeier, in Realistic Input for Realistic Images. ACM Press (ACM SIGGRAPH 95 Course Notes, 1995). Also appeared in the ACM SIGGRAPH 98 Course Notes - A Basic Guide to Global Illumination

26. P. Callet, Pertinent data for modelling pigmented materials in realistic rendering, Computer Graphics Forum 15(2), 119 (1996)

27. T. Nishita, Y. Dobashi, in Proceedings of Computer Graphics International 2001 (IEEE Computer Society, 2001), pp. 149-156

28. J.R. Frisvad, N.J. Christensen, H.W. Jensen, Computing the scattering properties of participating media using Lorenz-Mie theory, ACM Transactions on Graphics 26(3) (2007). Article 60

29. L.G. Henyey, J.L. Greenstein, Diffuse radiation in the galaxy, Annales d'Astrophysique 3, 117 (1940). Also in The Astrophysical Journal 93, 1941

30. C.F. Bohren, D.P. Gilra, Extinction by a spherical particle in an absorbing medium, Journal of Colloid and Interface Science 72(2), 215 (1979)

31. H.C. van de Hulst, Light Scattering by Small Particles (John Wiley \& Sons, Inc., New York, 1957). Unabridged and corrected version of the work published by Dover Publications, Inc., in 1981

32. L. Lorenz, Lysbevægelser i og uden for en af plane Lysbølger belyst Kugle, Det kongelig danske Videnskabernes Selskabs Skrifter 6(1), 2 (1890). 6. Række, naturvidenskabelig og mathematisk Afdeling

33. G. Mie, Beiträge zur Optik trüber Medien, speziell kolloidaler Metallösungen, Annalen der Physik 25(3), 377 (1908). IV. Folge

34. M. Kerker, The Scattering of Light and Other Electromagnetic Radiation (Academic Press, New York, 1969)

35. J.V. Dave, Scattering of electromagnetic radiation by a large, absorbing sphere, IBM Journal of Research and Development 13(3), 302 (1969)

36. C.F. Bohren, D.R. Huffman, Absorption and Scattering of Light by Small Particles (John Wiley \& Sons, Inc., 1983)

37. G.W. Kattawar, G.N. Plass, Electromagnetic scattering from absorbing spheres, Applied Optics 6(8), 1377 (1967) 
38. W.J. Wiscombe, Improved Mie scattering algorithms, Applied Optics 19(9), 1505 (1980)

39. W.C. Mundy, J.A. Roux, A.M. Smith, Mie scattering by spheres in an absorbing medium, Journal of the Optical Society of America 64(12), 1593 (1974)

40. V.E. Cachorro, L.L. Salcedo, New improvements for Mie scattering calculations, Journal of Electromagnetic Waves and Applications 5(9), 913 (1991)

41. D.W. Mackowski, R.A. Altenkirch, M.P. Menguc, Internal absorption cross sections in a stratified sphere, Applied Optics 29(10), 1551 (1990)

42. Z.S. Wu, Y.P. Wang, Electromagnetic scattering for multilayered sphere: Recursive algorithms, Radio Science 26(6), 1393 (1991)

43. W. Yang, Improved recursive algorithm for light scattering by a multilayered sphere, Applied Optics 42(9), 1710 (2003)

44. H.C. van de Hulst, On the attenuation of plane waves by obstacles of arbitrary size and form, Physica 15(8-9), 740 (1949)

45. J. Randrianalisoa, D. Baillis, L. Pilon, Modeling radiation characteristics of semitransparent media containing bubbles or particles, Journal of the Optical Society of America A 23(7), 1645 (2006)

46. J. Yin, L. Pilon, Efficiency factors and radiation characteristics of spherical scatterers in an absorbing medium, Journal of the Optical Society of America A 23(11), 2784 (2006)

47. Q. Fu, W. Sun, Apparent optical properties of spherical particles in absorbing medium, Journal of Quantitative Spectroscopy and Radiative Transfer 100(1-3), 137 (2006)

48. T.C. Grenfell, S.G. Warren, Representation of a nonspherical ice particle by a collection of independent spheres for scattering and absorption of radiation, Journal of Geophysical Research 104(D24), 31,697 (1999)

49. S.P. Neshyba, T.C. Grenfell, S.G. Warren, Representation of a non-spherical ice particle by a collection of independent spheres for scattering and absorption of radiation: 2. Hexagonal columns and plates, Journal of Geophysical Research 108(D15, 4448), 6 (2003)

50. T.C. Grenfell, S.P. Neshyba, S.G. Warren, Representation of a non-spherical ice particle by a collection of independent spheres for scattering and absorption of radiation: 3 . Hollow columns and plates, Journal of Geophysical Research 110(D17203), 1 (2005)

51. J.R. Frisvad, Light, Matter, and Geometry: The Cornerstones of Appearance Modelling (VDM (Verlag Dr. Müller), 2008)

52. J.A. Lock, G. Gouesbet, Generalized Lorenz-Mie theory and applications, Journal of Quantitative Spectroscopy \& Radiative Transfer 110(11), 800 (2009). Review

53. G. Gouesbet, Generalized Lorenz-Mie theories, the third decate: A perspective, Journal of Quantitative Spectroscopy \& Radiative Transfer 110(14-16), 1223 (2009). Review

54. E.P. Lafortune, Y.D. Willems, in Proceedings of the 7th Eurographics Workshop on Rendering (1996), pp. 91-100

55. H.D. Goff, A.R. Hill, in Dairy Science and Technology Handbook: Principles and Properties, vol. 1, ed. by Y.H. Hui (VCH Publishers, Inc., New York, 1993), chap. 1, pp. 1-81

56. P.F. Fox, P.L.H. McSweeney, Dairy Chemistry and Biochemistry (Blackie Academic \& Professional, London, 1998)

57. X. Quan, E.S. Fry, Empirical equation for the index of refraction of seawater, Applied Optics 34(18), 3477 (1995)

58. P.D.T. Huibers, Models for the wavelength dependence of the index of refraction of water, Applied Optics 36(16), 3785 (1997)

59. H. Du, R.C.A. Fuh, J. Li, L.A. Corkan, J.S. Lindsey, PhotochemCAD: A computer-aided design and research tool in photochemistry, Photochemistry and Photobiology 68(2), 141 (1998)

60. J. Koziol, Studies on flavins in organic solvents I: Spectral characteristics of riboflavin, riboflavin tetrabutyrate and lumichrome, Photochemistry and Photobiology 5, 41 (1966)

61. G.M. Hale, M.R. Querry, Optical constants of water in the $200-\mathrm{nm}$ to $200-\mu \mathrm{m}$ wavelength region, Applied Optics 12(3), 555 (1973)

62. M.C. Michalski, V. Briard, F. Michel, Optical parameters of milk fat globules for laser light scattering measurements, Lait 81, 787 (2001)

63. P. Walstra, R. Jenness, Dairy Chemistry and Physics (John Wiley \& Sons, New York, 1984) 
64. P. Walstra, Effect of homogenization on the fat globule size distribution in milk, Netherland's Milk Dairy Journal 29, 279 (1975)

65. D.W. Olson, C.H. White, R.L. Richter, Effect of pressure and fat content on particle sizes in microfluidized milk, Journal of Dairy Science 87(10), 3217 (2004)

66. R. Attaie, R.L. Richtert, Size distribution of fat globules in goat milk, Journal of Dairy Science 83, 940 (2000)

67. R. Gebhardt, W. Doster, J. Friedrich, U. Kulozik, Size distribution of pressure-decomposed casein micelles studied by dynamic light scattering and AFM, European Biophysics Journal 35, 503 (2006)

68. D.G. Schmidt, P. Walstra, W. Buchheim, The size distribution of casein micelles in cow's milk, Netherland's Milk Dairy Journal 27, 128 (1973)

69. A. Stockman, L.T. Sharpe, The spectral sensitivities of the middle- and long-wavelengthsensitive cones derived from measurements in observers of known genotype, Vision Research 40(13), 1711 (2000) 